\title{
Microspore-derived Embryo Formation and Morphological Changes during the Isolated Microspore Culture of Radish (Raphanus sativus L.)
}

\author{
NaRae $\mathrm{Han}^{1 \dagger}$, Sung Un $\mathrm{Kim}^{2 \dagger}$, Han Young Park ${ }^{3}$, and Haeyoung $\mathrm{Na}^{1,2^{*}}$ \\ ${ }^{1}$ Nature Resource Institute, Mokpo National University, Muan 534-729, Korea \\ ${ }^{2}$ Department of Horticultural Science, Mokpo National University, Muan 534-729, Korea \\ ${ }^{3}$ Department of Bioresource Engineering, Sejong University, Seoul 143-747, Korea
}

\begin{abstract}
Raphanus sativus L. cv. Taebaek, a efficiently microspore-derived embryo (MDE)-forming cultivar, and 'Chungwoon', a non-MDE-forming cultivar were selected as donor plants for isolated microspore culture. Radish flower bud of 2.0 (small, S), 4.0 (medium, M), and 6.0 (large, $\mathrm{L}$ ) $\pm 0.5 \mathrm{~mm}$ in length were isolated to determine the temporal relationship between flower bud size and MED yield. Anatomical observations revealed no difference in the structure of the flower buds between the two cultivars. In both cultivars, the stigmas were much longer than the floral leaf in M-sized flower buds. The MDE yields for 'Taebaek' per petri dish were 6.6 and 1.3 for M- and L-sized of flower buds, respectively, but MDE formation was not induced in the S flower buds. On the other hand, 'Chungwoon' failed to form MDEs in all flower buds. The microspore density of 'Taebaek' was 1.3 times more than that of 'Chungwoon' for M sized flower buds. Of the M-sized buds from 'Taebaek' and 'Chungwoon', 92.1 and $81.6 \%$, respectively, were in the late uninucleate microspore stage, which is characterized by the highest frequency of MDE formation. Anatomical observations of MDE formation revealed that the microspores were able to divide to form a primordium from which cell division took place continuously in the 'Teabeak' cultivar. However, the microspores of 'Chungwoon' failed to progress beyond the primodium stage, resulting in lack of MDE formation. By contrast, after the formation of the primordium, various developmental stages of embyos from microspore were observed in the 'Taebaek' cultivar. These results can be used to determine MDE forming potentials of radish cultivars.
\end{abstract}

Additional key words: anatomical observation, developmental stage, embryogenesis, flower bud, late uninucleate stage

\section{Introduction}

Radish (Raphanus sativus L.) is one of the most popular root and leaf vegetables in East Asia. It is a cruciferous crop that is used as both a medicinal and edible plant. Nutritionally, radish is a rich source of vitamins $\mathrm{B}$ and $\mathrm{C}$, peroxidase, and organic isothiocyanates, and for this reason radish has been used for many medicinal purposes (Curtis, 2007). Breeding practices such as mass selection, pedigree selection, and bud pollination have been used to increase yield and to improve the plant's medicinal value. However, conventional breeding takes a long time, so genetic engineering has emerged as an important technique for producing improved varieties of many plants (Curtis, 2011).

Isolated and in-vitro cultured microspores can divide repeatedly and develop into embryos. The production of doubled haploid plants by embryogenesis is a useful method for obtaining homozygous individuals in a short period of time because it does not require the numerous steps involved in conventional breeding programs (Babbar et al., 2004; Forster et al., 2007; Seguí-Simarro and Nuez, 2008; Takahashi et al., 2011; Touraev et al., 2001). In many plants, the frequency of embryo induction is not high enough to perform molecular and biochemical characterization, except

\footnotetext{
*Comesponding author: somerze@mokpo.ac.kr

${ }^{\dagger}$ These authors contributed equally to this work.

※ Received 2 November 2013; Revised 16 December 2013; Accepted 5 February 2014. This work was supported by a grant from the NextGeneration BioGreen 21 Program (Plant Molecular Breeding Center no. PJ009100), Rural Development Administration, Republic of Korea. 
for a few species such as rice (Cho and Zapata, 1990; Raina and Irfan, 1998; Xie et al., 1995, 1997), tobacco (HeberleBors, 1989; Touraev et al., 1996a, 1996c; Žárský et al, 1992, 1995), and oilseed rape (Chuong et al., 1988; Cloutier et al., 1995; Gu et al., 2004; Polsoni et al., 1988; Senratna et al., 1991). In addition, different cultivars within a same species that can generate microspore-derived embryos (MDEs) show different MDE efficiency. For this reason, microspore culture is difficult to apply in some crops. Numerous factors affect a positive embryogenic response, including genotype and the growth conditions of the donor plants, the developmental stage of the flower buds or microspores, pretreatment of the flower buds, and the culture medium. Among these factors, the genotype of the donor plant and the developmental stage of the microspores are particularly important (Babbar et al., 2004; Seguí-Simarro and Nuez, 2008; Smykal, 2000). The effect of donor plant genotype was previously reported for several crucifer species (Babbar et al., 2004), but the focus of that study was to select a variety that had the ability to induce MDE formation.

Microspores of cruciferous crops at a specific stage of development are responsive to MDE formation. A previous report suggested that the greatest response to embryogenesis is observed when microspores are isolated at the first pollen mitosis from the late uninucleate to the early binucleate stage (Binarova et al., 1997; Takahata et al., 1991; Touraev et al., 1996a, 1996b). The role of the developmental stage of pollen at the moment of culture initiation, however, is still undetermined. The developmental stage of microspore varies greatly depending on the cultivar or species, and it is difficult to determine the developmental stage by microscopy. Therefore, it is necessary to select flower buds containing microspores that have the ability to induce MDE formation. The developmental stage of a microspore is associated with the length of the flower bud, floral leaf, or their ratio (Babbar et al., 2004; Smykal, 2000; Weber et al., 2005). Up until now, flower buds with a longer stigma length than to that of the floral leaf have been chosen from isolated microspore cultures of cruciferous crops (Chun et al., 2011a, 2011b). However, this method is only applicable to a few varieties. Therefore, the best time to separate the microspore should be investigated to elucidate the mechanism of non-MDE formation.

The objective of this study was to show a correlation between the length or structure of a flower bud and the microspore developmental stage in $R$ sativus $\mathrm{L}$. In addition, the factors involved in preventing embryogenesis were investigated through the observation of MDEs during culture using microscopy.

\section{Materials and Methods}

\section{Plant Materials}

Raphanus sativus L. cvs. Taebaek and Chungwoon (Monsanto Co., Seoul, Korea) were used as donor plants for isolated microspore culture. Seeds of the donor plants were sown in 50 hole plugs. When the seedlings were 5 weeks old and showing 2-3 leaves, floral differentiation was induced in a cold room at $5.0 \pm 1.0^{\circ} \mathrm{C}$ under continuous light for 4 weeks. After floral differentiation and the beginning of generative development, the plants were transferred to a greenhouse (length $\times$ width $\times$ height $=3.0 \times 7.0 \times 3.0$ $\mathrm{m}^{3}$ ) which was equipped with an air-conditioning and heating system to maintain a day/night temperature between $23.0 / 16.0 \pm 1.0^{\circ} \mathrm{C}$.

\section{Observation of Flower Bud Structure}

Pre-blossom buds were collected and classified into three groups based on their length as follows: 2.0 (small, S), 4.0 (medium, M), and 6.0 (large, $\mathrm{L}$ ) $\pm 0.5 \mathrm{~mm}$. The floral leaf, anther, and stigma were examined using a microscope (Demis-ME; Siwon Optical Technology Co., Ltd., Anyang, Korea).

\section{Technique for Isolated Microspore Culture}

Those flower buds ( $4.0 \pm 0.5 \mathrm{~mm}$ in length) with a longer stigma than the length of the floral leaf were collected. The buds were sterilized in 1\% sodium hypochlorite for 15 min followed by rinsing three times with sterile deionized water for $3 \mathrm{~min}$. The buds were then ground in a mortar containing B5 liquid medium (Gamborg et al., 1968) supplemented with $13 \%$ sucrose. A microspore suspension was obtained by filtration through $45-\mu \mathrm{m}$ nylon screens. This suspension was centrifuged three times at $1,000 \mathrm{rpm}$ for $3 \mathrm{~min}$. After washing, the microspores were suspended in Nitsch and Nitsch (NLN) liquid medium (Lichter, 1982) supplemented with $15 \%$ sucrose. The microspore suspension $(2.5 \mathrm{~mL})$ was placed in a $60 \times 15 \mathrm{~mm}$ sterile petri dish, and $0.1 \mathrm{~mL}$ of $1 \%$ activated charcoal was dropped onto the petri dish, which was subsequently sealed with parafilm.

B5-13\% and NLN-15\% media were adjusted to a $\mathrm{pH}$ of 6.0 and $\mathrm{pH} 5.8$, respectively, using $\mathrm{NaOH}$, and were filter-sterilized using a $25 \mu \mathrm{m}$ low protein-binding membrane filter (Corning, NY, USA). The microspores were incubated in darkness at $32.5^{\circ} \mathrm{C}$ for 2 days prior to maintenance at $25.0^{\circ} \mathrm{C}$. 
After 17 days of culture in the dark, the petri dish was transferred to a gyratory shaker and agitated at $70 \mathrm{rpm}$ and $25.0^{\circ} \mathrm{C}$.

The cotyledonary embryos were transferred directly to B5 medium supplemented with 3\% sucrose and $8 \%$ agar. All MDEs were incubated to induce shoot and root regeneration. Germinated plants were transferred to artificial soil.

\section{Microspore Culture for MDE Formation}

Flower buds were collected and classified into three groups based on their length: $2.0(\mathrm{~S}), 4.0$ (M), 6.0 (L) \pm $0.5 \mathrm{~mm}$. The embryo yield was examined 4 weeks after microspore isolation. The embryo yield was the mean embryo number in ten petri dishes.

\section{Microspore Density}

The number of microspores was counted using a disposable hemocytometer (C-Chip DHC-NO1-5, Incyto Co., Ltd., Cheonan, Korea). A microspore with liquid medium $(10 \mu \mathrm{L})$ was loaded into the injection area, and then the number of the microspores was counted under a microscope. The hemocytometer consisted of 9 squares, each measuring $1.0 \times 1.0 \mathrm{~mm}^{2}$, and the depth of the chamber was $0.1 \mathrm{~mm}$. Each square had a total volume of $10^{-4} \mathrm{~mL}$ (length $\times$ width $\times$ height $=0.1 \times 0.1 \times 0.01 \mathrm{~cm}^{3}=10^{-4} \mathrm{~cm}^{3}=10^{-4} \mathrm{~mL}$ ). Therefore, the original number of microspores needed to be multiplied by $10^{4}$.

\section{Observation of the Microspores Developmental Stage}

The developmental stage of the microspores was traced by fluorescence microscopy using 4',6-diamidino-2-phenylindole (DAPI) staining. The developmental stages of the microspores were assigned as follows: early uninucleate, late uninucleate, and binucleate (Fig. 6). In the uninucleate stage, the microspores contained one nucleus. In the early and late uninucleate stages, the nucleus of the microspore was located at the center and off, respectively. In the binucleate stage, the microspores contained two nuclei.

DAPI is a fluorescent stain that binds preferentially to A-T rich regions of DNA. It has been used to stain both fixed and live cells. When bound to DNA, DAPI has an absorption maximum at a wavelength of $358 \mathrm{~nm}$ (Tarnowski et al., 1991). DAPI was dropped directly onto the NLN medium containing microspores. The medium was then incubated in the dark at room temperature. After $1 \mathrm{~h}$, a staining sample was rinsed several times and the nucleus was observed by a confocal laser scanning microscopy (TCS SP5 ADBS/Tandem; Leica Microsystems Co., Wetzlar,
Germany). Image processing was carried out using a resonant scanner with an emission spectral range of 425-475 $\mathrm{nm}$. The DAPI/DNA complex was excited with a 405-nm laser, and the measured range was $119.2 \times 119.2 \mathrm{~nm}^{2}$.

\section{Anatomical Observation of MDE Development}

MDE development from the microspores was observed at intervals of 2 days for 30 days. The structural pattern of the microspores was observed soon after isolation using an optical microscope (Axioskop2 Plus; Carl Zeiss Co., Thornwood, NY, USA). MDEs generated from the microspores of the 'Taebaek' cultivar were observed using a microspore (Demis-ME; Siwon Optical Technology Co., Ltd., Anyang, Korea) 20 days after the culture was isolated.

\section{Statistical Analysis}

All results were replicated ten times. A statistical analysis was performed with SigmaPlot 12.0 statistical software. The values are presented as the average of a replicate analysis in each of the experiments followed by the standard error.

\section{Result and Discussion}

The size and ratio of the floral leaf, stamen, and stigma within the flower buds of the 'Taebaek' and 'Chungwoon' cultivars were examined by microscopy (Fig. 1). In both cultivars, the stigma was invisible since the length of the stamen was longer than that of the stigma in the S-sized flower buds. In the M-sized flower buds, the length of the stigma was longer than that of the stamen and floral leaf. The stigma was not observed in the L-sized flower buds because it was located under the floral leaf. The structure of the floral leaf, anther, and stigma according to flower bud size showed obvious differences in length and ratio, while there was no difference in the structures between the two cultivars.

The M-sized flower buds had the highest number of microspores in the 'Taebaek' and 'Chungwoon' cultivars, followed by the L- and S-sized flower buds (Figs. 2 and 3). Furthermore, the number of microspores in the M-sized flower buds was higher in 'Taebaek' than that of 'Chungwoon', which indicated a high efficiency of MDE formation compared with 'Chungwoon', which cannot induce MDEs.

The response MDE formation rate was investigated to select the optimum bud size for isolated microspore culture. The MDE yields per petri dish in the 'Taebaek' cultivar were 6.6 and 1.3 for the M- and L-sized flower buds, respectively 

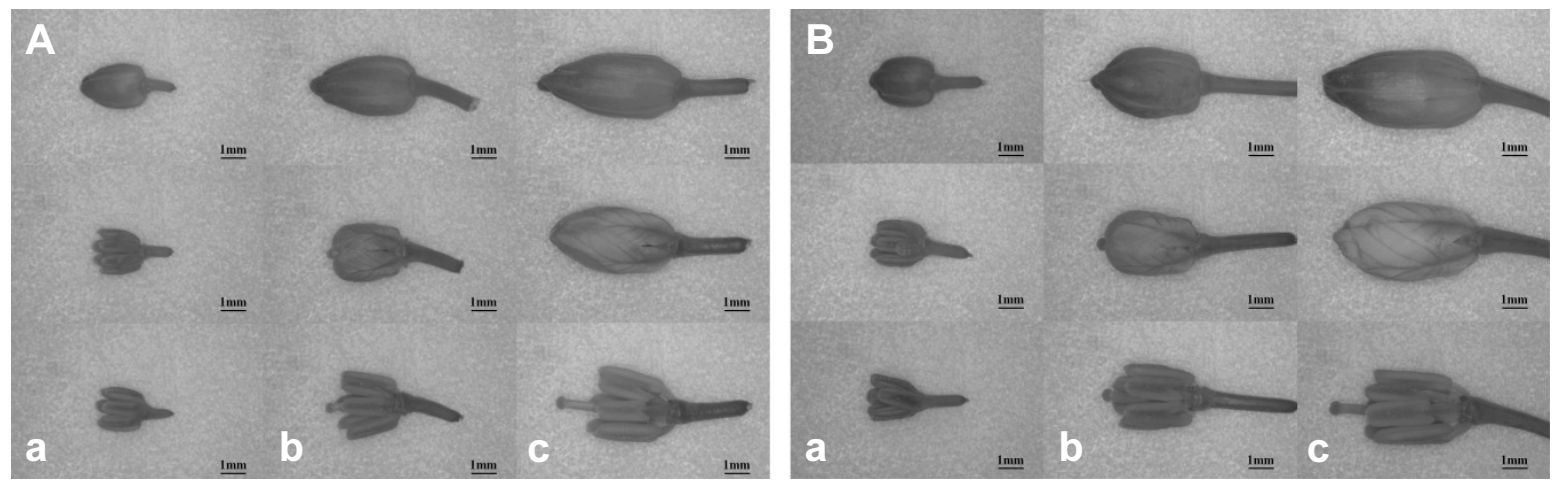

Fig. 1. Structure of the flower buds, floral leaves, anthers, and stigmas in the 'Taebaek' (A) and 'Chungwoon' (B) cultivars by flower bud length. a, $2.0 \pm 0.5 \mathrm{~mm}$; b, $4.0 \pm 0.5 \mathrm{~mm}$; c, $6.0 \pm 0.5 \mathrm{~mm}$.

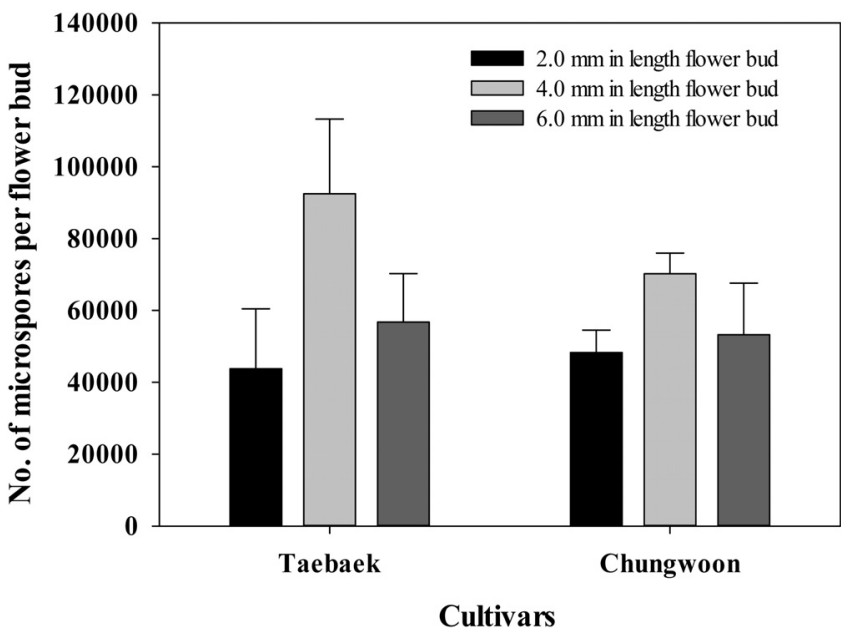

Fig. 2. The number of microspores per flower bud in 'Taebaek' and 'Chungwoon' cultivars according to the flower bud size. Data were obtained from 10 replicates of the flower bud with standard error of the mean.

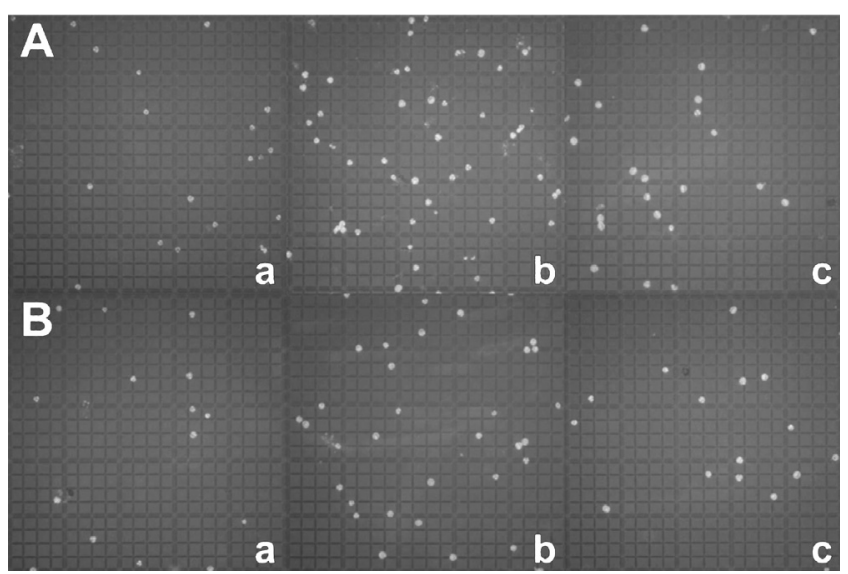

Fig. 3. Microspore density in the 'Taebaek' (A) and 'Chungwoon' (B) cultivars according to the flower bud size. a, $2.0 \pm 0.5$ $\mathrm{mm} ; \mathrm{b}, 4.0 \pm 0.5 \mathrm{~mm} ; \mathrm{c}, 6.0 \pm 0.5 \mathrm{~mm}$.

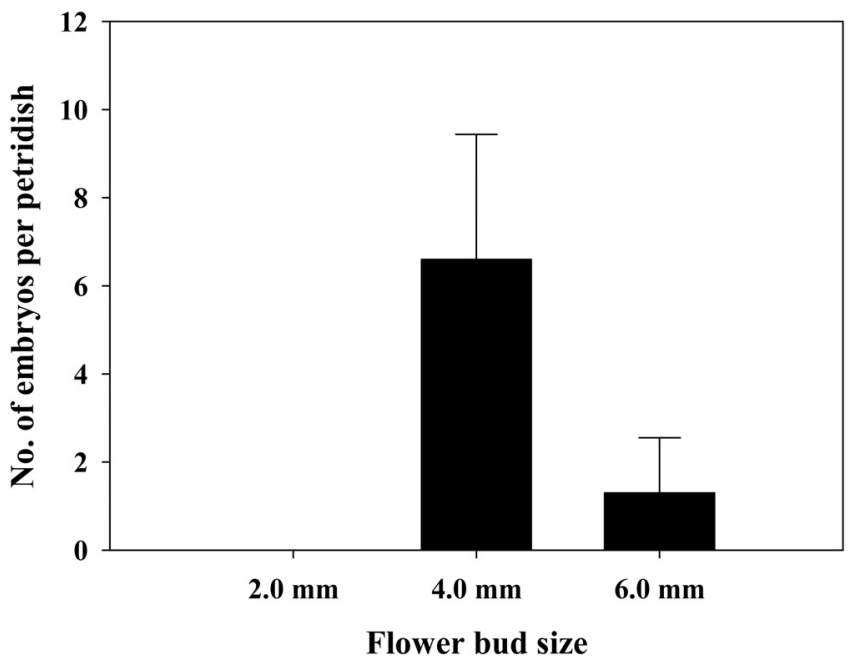

Fig. 4. Yield of microspore-derived embryos (no. of microsporederived embryos per petri dish) in the 'Taebaek' cultivar according to the flower bud size. The data were collected 4 weeks after culturing. The values given the average of ten replicate analyses in each of the experiments followed by standard error.

(Figs. 4 and 5). However, MDEs were not induced in the S-sized flower bud. On the other hand, 'Chungwoon' failed to form MDEs in S-, M-, and L-sized of flower buds.

A total of 82.1 and $81.6 \%$ of the microspores were in the late uninucleate stage in M-sized flower buds of 'Taebaek' and 'Chungwoon', respectively. The M-sized flower buds contained the greatest number of late uninucleate microspores (Table 1 and Fig. 6). The development of the microspores varied with flower bud size.

Flower bud length is a simple parameter to use in selecting flower buds containing a microspore with the ability to induce an MDE (Babbar et al., 2004; Smykal, 2000). Various approaches to induce MDEs in R. sativus L. have been 


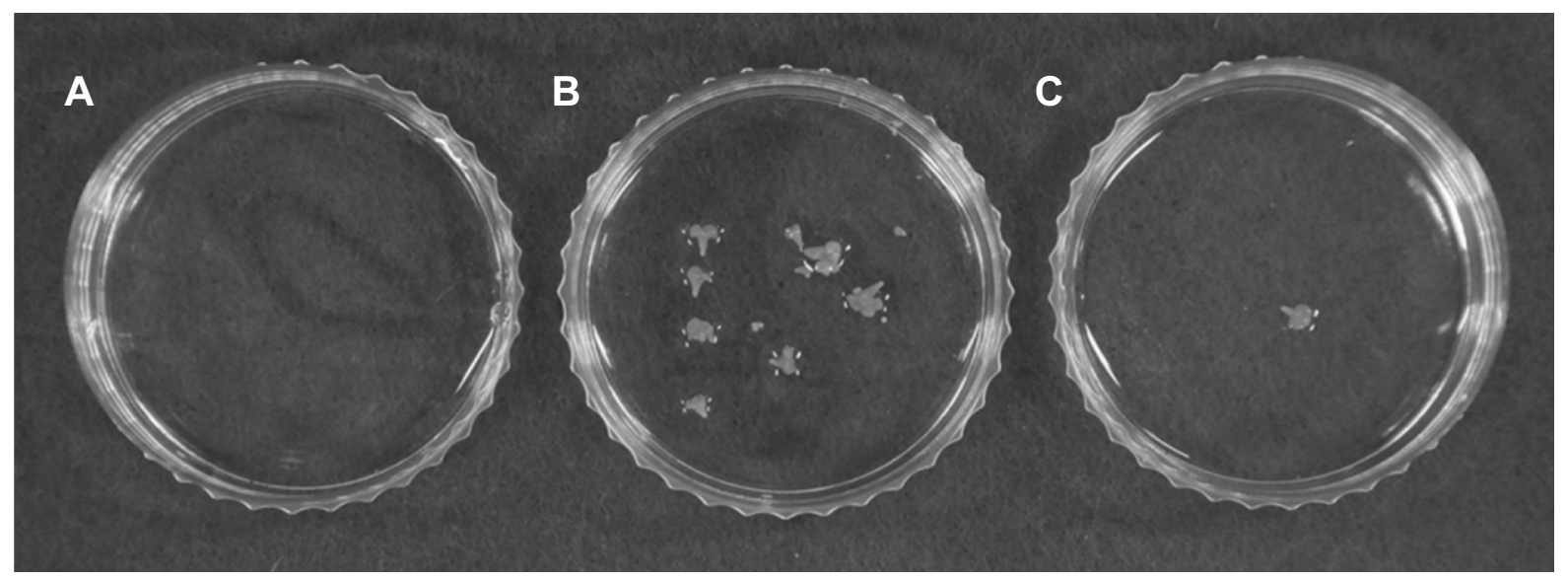

Fig. 5. Microspore-derived embryos of the 'Taebaek' cultivar according to the flower bud size. A, $2.0 \pm 0.5 \mathrm{~mm}$; $\mathrm{B}, 4.0 \pm 0.5$ $\mathrm{mm}$; C. $6.0 \pm 0.5 \mathrm{~mm}$.

Table 1. Percentage of microspores at each developmental stage in the 'Taebaek' and 'Chungwoon' cultivars.

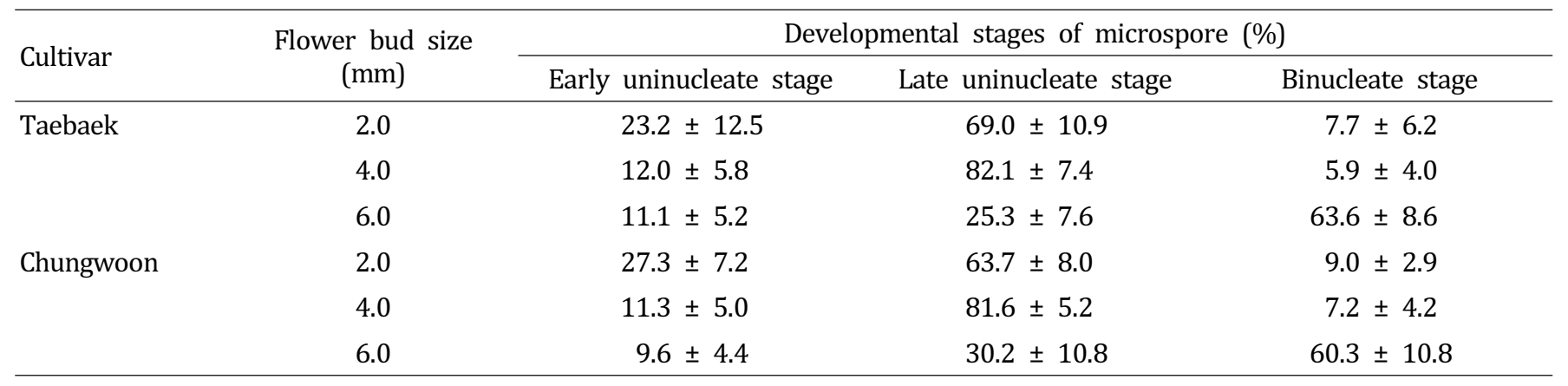

reported (Chun et al., 2011a, 2011b; da Silva Dias 1999; Takahata et al., 1996). Chun et al. (2011b) observed that the highest MDE yield was obtained when the microspore was isolated from buds that were $2.5-4.5 \mathrm{~mm}$ in length. A flower bud showing a longer length of stigma compared to that of the floral leaf is considered the standard for separating the microspore in cruciferous crops (Babbar et al., 2004; Chun et al., 2011a, 2011b; Smykal, 2002). The results from those studies are consistent with our finding of MDE formation according to the length of the flower bud. The M-sized of flower buds had the highest microspore content and showed the highest MDE yield in 'Taebaek'. It is assumed that the microspore content of a flower bud is likely to affect MDE formation. Previous reports showed that a microspore has totipotency during the late uninucleate to early binucleate stages (Binarova et al., 1997; Touraev et al., 1996a, 1996b). Therefore, the selection of a flower bud containing microspores at the optimal stage is an important factor to increase rates of embryogenesis. Our results show that peak MDE formation in 'Taebaek' occurred when microspores were isolated from M-sized flower buds, which had the highest content of late uninucleate stage microspores. These results are in agreement with those of previous studies of cruciferous crops in which MDEs were obtained from microspores at the late uninucleate stage (Baillie et al., 1992; Burnett et al., 1992; Hansen and Svinnset, 1993; Telmer et al., 1992). This study found a conclusion about the correlation between the length of the flower bud and the efficiency of MDE formation based on the fact that the MDE yield was highest in M-sized flower buds, which showed high contents of late uninucleate stage microspores. Thus, our results can be applied to the isolated microspore culture of $R$. sativus $\mathrm{L}$.

The processes in MDE development from a microspore are shown in Fig. 7. At the time of isolated culture, the microspore was about $10.0 \pm 1.0 \mu \mathrm{m}$ in diameter in the 'Taebaek' and 'Chungwoon' cultivars. After 10 days in microspore isolation, some microspores had begun to break out of the microspore wall. The microspore then divided to form a primordium, and continuous cell division occurred 
from the primordium in both cultivars. However after that, the microspores did not develop as MDEs, and cell division did not proceed in the 'Chungwoon' cultivar (Fig. 7B).

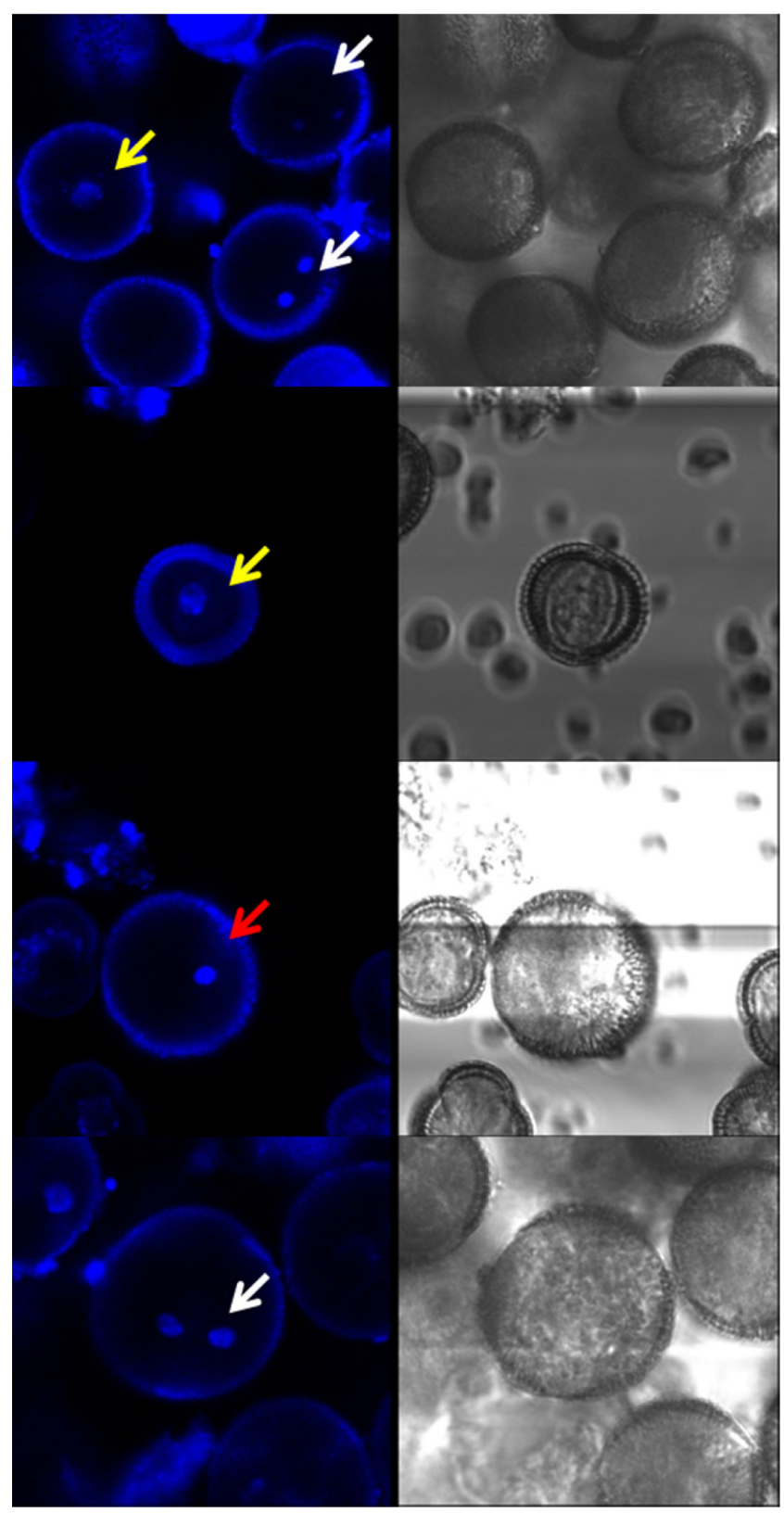

Fig. 6. Microspore developmental stages in $R$ sativus L. Yellow, red, and white arrows indicated early uninucleate, late uninucleate, biuninucleate stage of microspores, respectively. The developmental stages of the microspores were traced by fluorescence microscopy using DAPI staining. Uninucleate microspores contained one nucleus. Early- and late-uninucleate microspore contained nuclei that were located in the center and off center, respectively. The binucleate microspore contained two nuclei.
Furthermore, the microspore was far too small to be seen by the human eye in the 'Chungwoon' cultivar. On the other hand, after the occurrence of the primordium, various developmental stages beginning from the microspore can be observed in the 'Taebaek' cultivar (Fig. 7A). Similar observations of the microspore to embryo have been also reported for B. napus (Yeung, 2002; Yeung et al., 1996). However, anatomical observations of MDE formation process have not been reported for $R$. sativus $\mathrm{L}$. In general, MDE formation showed differences in response according to the genotype of the donor plant (Babbar et al., 2004; Smykal, 2002). It is too early to conclude that there is a distinct inherited difference according to the efficiency of MDE formation, since microspores isolated from 'Chungwoon' can be divided to form a primordium. It was found that the optimal environmental conditions for microspore to undergo embryo development after primordium formation differed between cultivars. A previous study of embryogenesis in cruciferous plants suggested that even though the frequency of MDE formation is low, it can be increased by changing the culture conditions. Temperature is a significant condition for MDE formation and further development (Binarova et al., 1997). The intensity of light and the photoperiod also affect the efficiency of MDE formation (Babbar et al., 2004). Furthermore, the frequency of MDE formation could be improved by the exchange of culture medium in some instances (Touraev, 1996c). Ethylene is one of the most important factors influencing the growth and development of microspores (Biddington, 1992; Wu et al., 2006). A previous study demonstrated that ethylene production inhibited callus development and shoot regeneration in plant tissue culture (Wu et al., 2006). The ethylene accumulation rate may have an effect on different developmental stages of microspores (Biddington, 1992; Gallie and Young, 2004). Further research is needed to understand the influence of ethylene. Therefore, the regulation of growth and culture conditions could be used to overcome varietal differences in MDE formation rate.

In conclusion, this study provides basic information for determining the optimum stage of bud development in order to isolate microspores based on the yield of MDEs, bud structure, microspore density, and microspore developmental stage. In addition, the developmental process during MDE formation was observed in $R$ sativus $\mathrm{L}$. This study provides the first anatomical observations of microspores isolated from $R$. sativus $\mathrm{L}$. Therefore, the results obtained in the present study may be applicable to isolated microspore cultures double haploid production in $R$. sativus $\mathrm{L}$. 

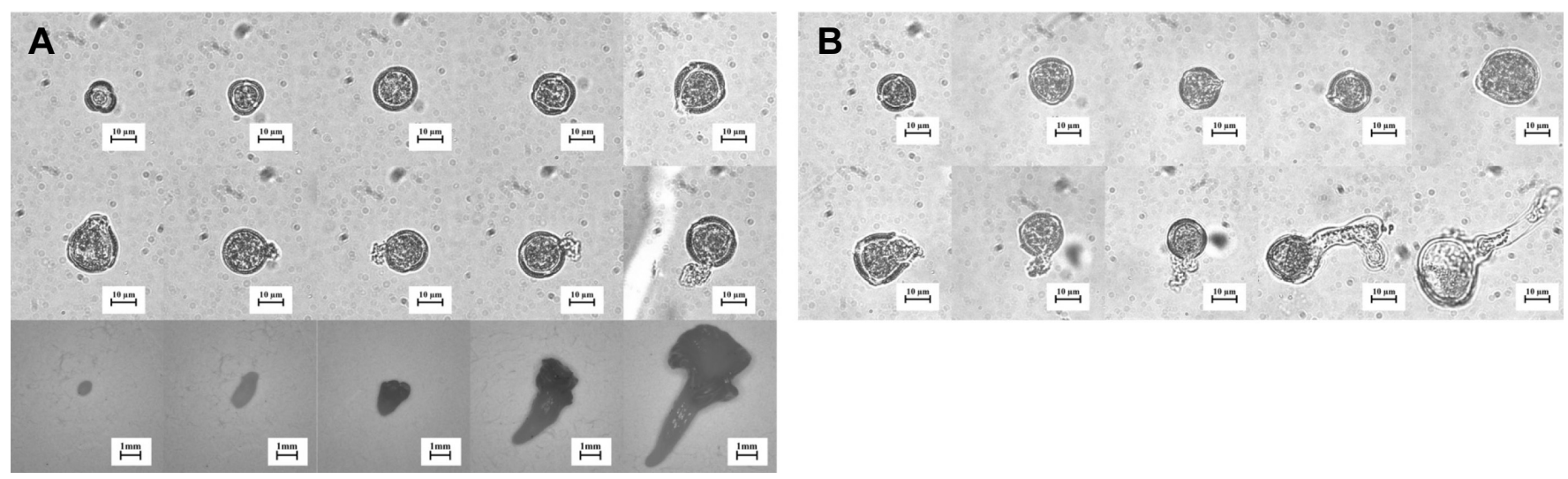

Fig. 7. Anatomical observations of the microspore developmental process in 'Taebaek' (A) and 'Chungwoon' (B) cultivars. Morphological changes in the microspores during cultivation in petri dish are shown. Pictures were taken every 2 days during 20 days of cultivation. Microspore-derived embryos generated from a microspore were observed in the 'Taebaek' cultivar.

\section{Literature Cited}

Babbar, S.B., P.K. Agarwal, S. Sahay, and S.S. Bhojwani. 2004. Isolated microspore culture of Brassica: An experimental tool for developmental. Indian J. Biotechnol. 3:185-202.

Baillie, A.M.R., D.J. Epp, D. Hutcheson, and W.A. Keller. In vitro culture of isolated microspores and regeneration of plants in Brassica campestris. Plant Cell Rep. 11:234-237.

Biddington, N.L. 1992. The influence of ethylene in plant tissue culture. Plant Growth Regulat. 11:173-187.

Binarova, P., G. Hause, V. Genklova, J.H.G. Bordewener, and M.M.L. Campagne. 1997. A short severe heat shock is required to induce embryogenesis in late bicellular pollen of Brassica napus L. Sexual Plant Reproduction 10:200-208.

Burnett, L., S. Yarow, and B. Huang. 1992. Embryogenesis and plant regeneration from isolated microspores of Brassica rapa L. ssp. oleifera. Plant Cell Rep. 11:215-218.

Cho, M.S. and F.J. Zapata. 1990. Plant regeneration from isolated microspore of Indica rice. Plant Cell Physiol. 31:881-885.

Chun, C. and H. Na. 2011a. Microspore-derived embryo formation in response to cold pretreatment, washing medium, and medium composition of radish (Raphanus sativus L.). Kor. J. Hort. Sci. Technol. 29:494-499.

Chun, C., H. Park, and H. Na. 2011b. Microspore-derived embryo formation in radish (Raphanus sativus L.) according the nutritional and environmental conditions. Hort. Environ. Biotechnol. 52: 530-535.

Chuong, P.V., C. Deslauriers, L.S. Kott, and W.D. Beversdorf. 1988. Effects of donor genotype and bud sampling on microspore culture of Brassica napus. Canadian J. Bot. 66:1653-1657.

Cloutier, C., M Cappadocia, and B.S. Landry. 1995. Study of microspore-culture responsiveness in oilseed rape (Brassica napus L.) by comparative mapping of a F2 population and two microspore-derived populations. Theoretical Appl. Genet. 91:841-847.
Curtis, I.S. 2007. Radish, p. 381-389. In: E.C.V. Pua and M.R. Davey (des.). Biotechnology in agriculture and forestry. Springer, Dordrecht.

Curtis, I.S. 2011. Genetic engineering of radish: Current achievements and future goals. Plant Cell Rep. 30:733-744.

da Silva Dias, J.C. 1999. Effect of activated charcoal on Brassica oleracea microspore culture embryogenesis. Euphytica 108:65-69.

Forster, B.P., E. Heberle-Bors, K.J. Kasha, and A. Touraev. 2007. The resurgence of haploids in higher plants. Trends Plant Sci. 12:368-375.

Gallie, D.R. and T.E. Young. The ethylene biosynthetic and perception machinery is differentially expressed during endosperm and embryo development in maize. Mol. Gen. Genomics 271: 267-281.

Gamborg, O.L., R.A. Miller, and K. Ojima. 1968. Nutrient requirements of suspension culture of soybean root cells. Exp. Cell Res. 50:151-158.

Gu, H.H., P. Hagberg, and W.J. Zhou. 2004. Cold pretreatment enhances microspore embryogenesis in oilseed rape (Brassica napus L.). Plant Growth Regulat. 42:137-143.

Hansen, M. and K. Svinnset. 1993. Microspore culture of swede (Brassica napus ssp. rapifera) and the effects of fresh and conditioned media. Plant Cell Rep. 12:496-500.

Heberle-Bors, E. 1989. Isolated pollen culture in tobacco: Plant reproductive development in a nutshell. Sexual Plant Reproduction 2:1-10.

Lichter, R. 1982. Induction of haploid plants from isolated pollen of Brassica napus Z. Pflanzenphysiol. 105:427-434.

Polsoni, L., L.S. Kott, and W.D. Beversdorf. 1988. Large-scale microspore culture technique for mutation-selection studies in Brassica napus. Canadian J. Bot. 66:1681-1685.

Raina, S.K. and S.T. Irfan. 1998. High-frequency embryogenesis and plantlet regeneration from isolated microspores of Indica rice. Plant Cell Rep. 17:957-962.

Seguí-Simarro, J.M. and F. Nuez. 2008. How microspores transform 
into haploid embryos: Changes associated with embryogenesis induction and microspore-derived embryogenesis. Physiol. Plant. 134:1-12.

Senratna, T., L. Kott, W.D. Beversdorf, and B.D. Mckersie. 1991. Desiccation of microspore derived embryos of oilseed rape (Brassica napus L.). Plant Cell Rep. 10:342-344.

Smykal, P. 2000. Pollen embryogenesis-The stress mediated switch from gametophytic to sporophytic development. Current status and future prospects. Biol. Plant. 43:481-489.

Takahata, Y., D.C.W. Brown, and W.A. Keller. 1991. Effect of donor plant age and inflorescence age on microspore culture of Brassica napus L. Euphytica 58:51-55.

Takahata, Y., H. Komatsu, and N. Kaizuma. 1996. Microspore culture of radish (Raphanus sativus L.): Influence of genotype and culture conditions on embryogenesis. Plant Cell Rep. 16:163-166.

Takahashi, Y., S. Yokoi, and Y. Takahata. 2011. Improvement of microspore culture method for multiple samples in Brassica. Breeding Sci. 61:96-98.

Tarnowski, B.I., F.G. Spinale, and J.H. Nicholson. 1991. DAPI as a useful stain for nuclear quantitation. Biotechnol. Histochem. 66:297-302.

Telmer, C.A., D.H. Simmonds, and W. Newcomb. 1992. Determination of developmental stage to obtain high frequencies of embryogenic microspores in Brassica napus. Physiol. Plant. 84:417-423.

Touraev, A., A. Ilham, O. Vicente, and E. Heberle-Bors. 1996a. Stress-induced microspore embryogenesis in tobacco: An optimized system for molecular studies. Plant Cell Rep. 15:561-565.

Touraev, A., A. Indrianto, I. Wratschko, O. Vicente, and E. HeberleBos. 1996b. Efficient microspore embryogenesis in wheat (Triticum aestivum L.) induced by starvation at high temperature. Sexual Plant Reproduction 9:209-215.

Touraev, A., M. Pfosser, O. Vicente, and E. Heberle-Bors. 1996c. Stress as the major signal controlling the developmental fate of tobacco microspores: towards a unified model of induction of microspore/pollen embryogenesis. Planta 200:144-152.

Touraev, A., M. Pfosser, and E. Heberle-Bors. 2001. The microspore: A haploid multipurpose cell. Advances Botanical Res. 35:53-109.

Weber, S., F. Unker, and W. Friedt. 2005. Improved doubled haploid production protocol for Brassica napus using microsproe colchicine treatment in vitro and ploidy determination by flow cytometry. Plant Breeding 124:511-513.

Wu, L.M., Y.M. Wei, and Y.L. Zheng. 2006. Effect of silver nitrate on the tissue culture of immature wheat embryos. Russian J. Plant Physiol. 53:530-534.

Xie, J., M. Gao, Q. Cai, X. Cheng, Y. Shen, and Z. Liang. 1995. Improved isolated microspore culture efficiency in medium with maltose and optimized growth regulator ombination in Japonica rice (Oryza sativa). Plant Cell. 42:245-250.

Xie, J.H., M.W. Gao, Z.Q. Liang, Q.Y. Shu, X.Y. Cheng, and Q.Z. Xue. 1997. The effect of cool-pretreatment on the isolated microspore culture and the free amino acid change of anthers in Japonica rice (Oryza sativa L). J. Plant Physiol. 151:79-82.

Yeung, E.C., M.H. Rahman, and T.A. Thorpe. 1996. Comparative development of zygotic and microspore-derived embryos in Brassica napus L. cv. Topas. I. histodifferentiation. Int. J. Plant Sci. 157:27-39.

Yeung, E.C. 2002. The canola microspore-derived embryo as a model system to study developmental processes in plants. J. Plant Biol. 45:119-133.

Žárský, V., D. Garrido, L. Říhová, J. Tupý, O. Vicente, and E. Heberle-Bors. 1992. Derepression of the cell cycle by starvation is involved in the induction of tobacco pollen embryogenesis. Sexual Plant Reproduction 5:189-194.

Žárský, V., D. Garrido, N. Eller, J. Tupy, O. Vicente, F. Schoffl, and E Heberle-Bors. 1995. The expression of a small heat shock gene is activated during induction of tobacco pollen embryogenesis by starvation. Plant Cell Environ. 18:139-147. 